\title{
Electronic Structure of Band-Tail Electrons in a Si:H
}

\author{
T. Umeda, ${ }^{1,2}$ S. Yamasaki, ${ }^{1, *}$ J. Isoya, ${ }^{1,3}$ A. Matsuda, ${ }^{4}$ and K. Tanaka ${ }^{1,2}$ \\ ${ }^{1}$ Joint Research Center for Atom Technology (JRCAT)-National Institute for Advanced Interdisciplinary Research (NAIR), \\ 1-1-4, Higashi, Tsukuba, 305, Japan \\ ${ }^{2}$ Institute of Materials Science, University of Tsukuba, Tsukuba, 305, Japan \\ ${ }^{3}$ University of Library and Information Science, 1-2, Kasuga, Tsukuba, 305, Japan \\ ${ }^{4}$ Electrotechnical Laboratory, 1-1-4, Umezono, Tsukuba, 305, Japan
}

(Received 1 July 1996)

\begin{abstract}
Electronic structures of the light-induced electron spin resonance (LESR) centers in undoped $a$-Si:H have been investigated by means of pulsed ESR techniques. Overlapping LESR signals of $g=2.004$ and 2.01 have been experimentally deconvoluted by using the difference in spin-lattice relaxation time between the two signals. The ${ }^{29} \mathrm{Si}$ hyperfine structures of the 2.004 signal clearly show that the wave function of this center spreads mainly over two Si atoms, which suggests that the origin of $g=2.004$ is electrons trapped at antibonding states of weak Si-Si bonds. [S0031-9007(96)01759-0]
\end{abstract}

PACS numbers: 71.55.Jv, 61.43.Dq

In hydrogenated amorphous silicon $(a-\mathrm{Si}: \mathrm{H})$, two different light-induced ESR (LESR) signals of $g=2.004$ and 2.01 have been detected at low temperatures and under illumination [1], in addition to the dark ESR signal of $g=2.0055$ that has been generally identified as a neutral dangling bond signal [2-4]. Street et al. [5] concluded, on the basis of the study of doping as well as photoluminescence experiments, that the LESR signals in undoped $a$-Si:H could be ascribed to conduction-band-tail electrons $(g=2.004)$ and valence-band-tail holes $(g=2.01)$. Yamasaki et al. [6] have succeeded in detecting hyperfine (hf) structures of ${ }^{29} \mathrm{Si}$ (a nuclear spin of $I=\frac{1}{2}$, natural abundance of 4.7 at. \%) of the LESR spectrum using a pulsed ESR technique, and discussed the nature of the LESR centers. However, due to the overlapping of two ESR signals of $g=2.004$ and 2.01 which are broadened by the random orientation and site-to-site variation of structures, the observed spectrum could not be deconvoluted into the two independent hf structures, which is necessary for more detailed arguments.

In this Letter, we present the first deconvoluted LESR spectra of $g=2.004$ and 2.01 signals in undoped $a-\mathrm{Si}: \mathrm{H}$, which were experimentally obtained by a combination of two different techniques; one is the deconvolution of the spectra by making use of the difference in a spinlattice relaxation time $\left(T_{1}\right)$ between two signals, and the other being pulsed ESR measurements on $a$-Si:H samples involving different amounts of ${ }^{29} \mathrm{Si}(9.1,4.7,1.6$ at .\%). By analyzing the ${ }^{29} \mathrm{Si}$ hf structures of the deconvoluted spectra of $g=2.004$ and 2.1 signals, it is demonstrated that LESR spin centers with $g=2.004$ originate from electrons trapped at the antibonding states of weak SiSi bonds.

Undoped $a$-Si:H samples were deposited by the rf-glow discharge technique from $\mathrm{SiH}_{4}$ with $\left[{ }^{29} \mathrm{Si}\right]=9.1,4.7$, and 1.6 at. $\%$ on a metal foil at a temperature of $250{ }^{\circ} \mathrm{C}$. Deposited flakes (about $1 \mu \mathrm{m}$ in thickness) were collected in quartz tubes for ESR measurements. The spin densities of those samples were in the range from $1 \times 10^{15}$ to $4 \times 10^{15} \mathrm{~cm}^{-3}$.

LESR spectra were determined by the technique of electron spin echo of pulsed ESR in order to obtain a wide-dynamic-range ESR signal [7]. In the echo-detected ESR technique the spectrum is obtained by recording the amplitude of the spin echo as a function of magnetic-field strength. In order to reduce the contribution of the $g=$ 2.0055 signal to a whole LESR signal, LESR spectra were measured at $30 \mathrm{~K}$ under illumination using a Ti-sapphire laser $(h \nu=1.7 \mathrm{eV}$, penetration depth for undoped $a-\mathrm{Si}: \mathrm{H}$ $\sim 10 \mu \mathrm{m}$, output power $=30 \mathrm{~mW} / \mathrm{cm}^{2}$ ). Under those conditions, the peak height of the LESR spectrum was more than 20 times larger than that of the dark ESR spectrum. We used a microwave frequency of $11 \mathrm{GHz}$ for the ESR measurements so that the overlapping of the 2.004 and 2.01 signals was reduced below the case of standard $X$-band $(\sim 9 \mathrm{GHz})$ measurements [8].

Figure 1 shows the LESR spectra of the samples with different contents of ${ }^{29} \mathrm{Si}$, which were determined by a twopulse Hahn echo-detected ESR technique $\left(90^{\circ}\right.$ pulse- $\tau$ $180^{\circ}$ pulse- $\tau$-echo) [7]. A repetition time (RT) of $10 \mathrm{~ms}$ of pulse sequences was used for ensuring a complete recovery of the spin system to the equilibrium state, by which the obtained spectra become equivalent to the absorption spectra of a conventional cw-ESR. As is seen in Fig. 1, base lines of the echo-detected ESR spectra are very flat because the echo signals can be picked up under a microwave-free condition, resulting in a wider dynamic range of the spectra than that of conventional cw-ESR spectra [7]. In the tail region, where the magnetic field is higher than $392.5 \mathrm{mT}$, a long tail is clearly observed, and its intensity increases with the increase of the ${ }^{29} \mathrm{Si}$ content of each sample. Actually, the integrated area of the tail structure of each sample was found to be proportional to the ${ }^{29} \mathrm{Si}$ content of each sample, indicating that the tail 


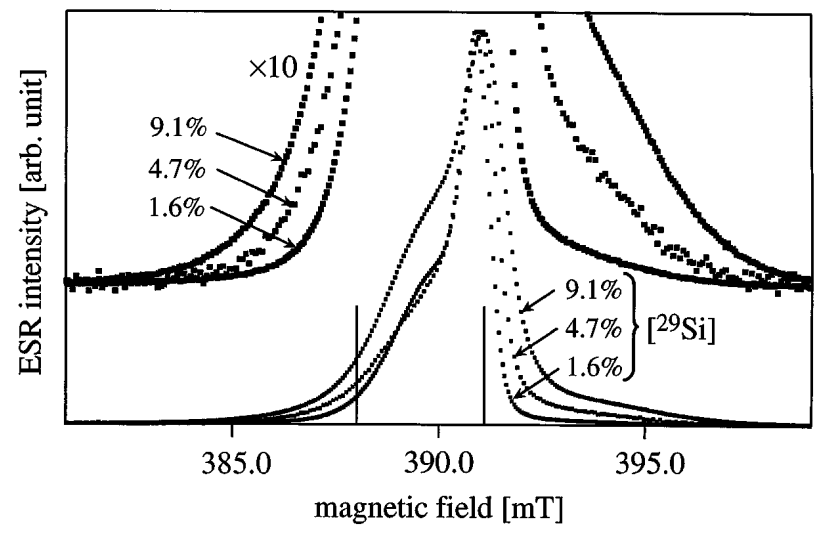

FIG. 1. LESR spectra at $30 \mathrm{~K}$ of undoped $a$-Si:H with $\left[{ }^{29} \mathrm{Si}\right]=9.1,4.7$, and 1.6 at. $\%$, which were determined by the two-pulse Hahn echo-detected ESR technique $(\tau=$ $240 \mathrm{~ns}, \mathrm{RT}=10 \mathrm{~ms}$ ). The spectra were normalized to their peak heights. Two vertical thin lines indicate the magnetic fields at which we have measured $T_{l}$.

structure on the higher-field side originates from ${ }^{29} \mathrm{Si} \mathrm{hf}$ interactions.

The spin-lattice relaxation curves of the LESR centers for the ${ }^{29} \mathrm{Si}$-enriched sample $\left(\left[{ }^{29} \mathrm{Si}\right]=9.1 \%\right)$ were directly measured by a pulse sequence of three-pulse inversion recovery $\left(180^{\circ}\right.$ pulse- $t-90^{\circ}$ pulse- $\tau-180^{\circ}$ pulse- $\tau$-echo; $t$ is scanned) at the magnetic field of 388.0 and $391.2 \mathrm{mT}$ corresponding to $g=2.004$ and 2.01 , respectively. The values of $T_{1}$ at $30 \mathrm{~K}$ for $g=2.004$ and 2.01 were determined to be $1.3 \mathrm{~ms}$ and $0.47 \mathrm{~ms}$, respectively. Thus, the $T_{1}$ value of $g=2.01$ is about one-third of that of $g=2.004$, which may be caused by a difference in electronic structure between the 2.01 and 2.004 spin centers.

When we use a RT of $0.5 \mathrm{~ms}$ for echo-detected ESR, the 2.01 signal becomes relatively stronger than the 2.004 signal simply because the 2.01 signal has a shorter recovery time (i.e., $T_{1}$ ). On the other hand, when we adopt the three-pulse stimulated echo $\left(90^{\circ}\right.$ pulse- $\tau-90^{\circ}$ pulse- $\tau^{\prime}-90^{\circ}$ pulse- $\tau$-echo, $\tau=240 \mathrm{~ns}, \tau^{\prime}=1 \mathrm{~ms}, \mathrm{RT}=$ $10 \mathrm{~ms}$ ) for measuring the echo-detected ESR, the 2.01 signal is relatively suppressed because $T_{1}$, in this case, gives the decay time of the echo signal with respect to $\tau^{\prime}$ [7], resulting in a faster decay of the 2.01 signal. In this way, we obtained two echo-detected ESR spectra with different relative intensities between 2.01 and 2.004 signals, which are shown in Fig. 2.

The two spectra in Fig. 2 were deconvoluted into two independent spectra by subtracting one from the other to cancel out either the 2.004 or the 2.01 signal alternatively, except for the region of strong overlap of the two signals where a deconvolution is difficult due to complexities such as $T_{1}$ distributions and cross relaxation. The result of deconvolution of the two spectra of Fig. 2 is shown in Figs. 3(a) and 3(b), respectively.

As is clearly seen in the figure, the hf structure observed on the high-field side belongs mainly to the $g=2.004$

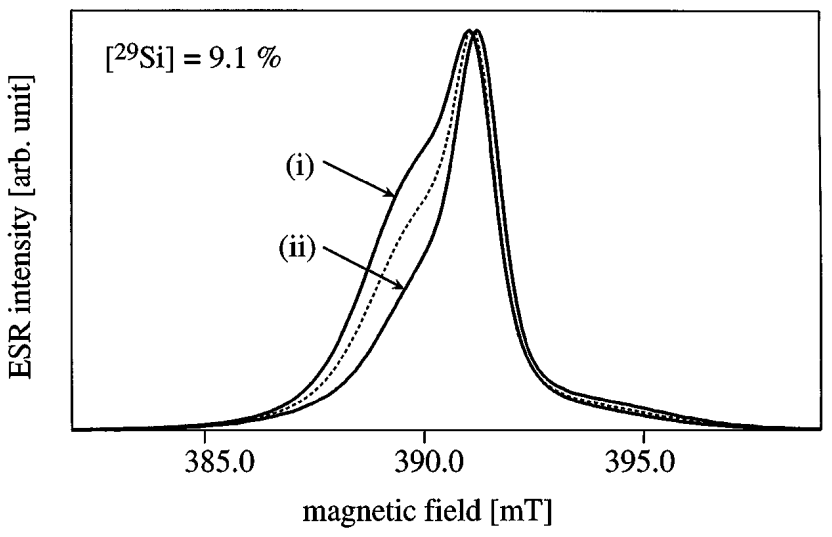

FIG. 2. LESR spectra of the ${ }^{29} \mathrm{Si}$-enriched sample, which were determined by echo-detected ESR using (i) two-pulse Hahn echo $(\tau=240 \mathrm{~ns}$, RT $=0.5 \mathrm{~ms})$ and (ii) three-pulse stimulated echo $\left(\tau=240 \mathrm{~ns}, \tau^{\prime}=1 \mathrm{~ms}\right.$, RT $\left.=0.5 \mathrm{~ms}\right)$. For comparison, the spectrum of the ${ }^{29} \mathrm{Si}$-enriched sample in Fig. 1 is also shown by a dashed line.

signal. The area fraction of the hf structure depends on the number of $\mathrm{Si}$ atoms, $N$, on which an electron spin is mainly located. The probability that all $N \mathrm{Si}$ atoms have no nuclear spin $\left({ }^{28} \mathrm{Si}\right.$ or $\left.{ }^{30} \mathrm{Si}\right)$ is $(1-p)^{N}$, where

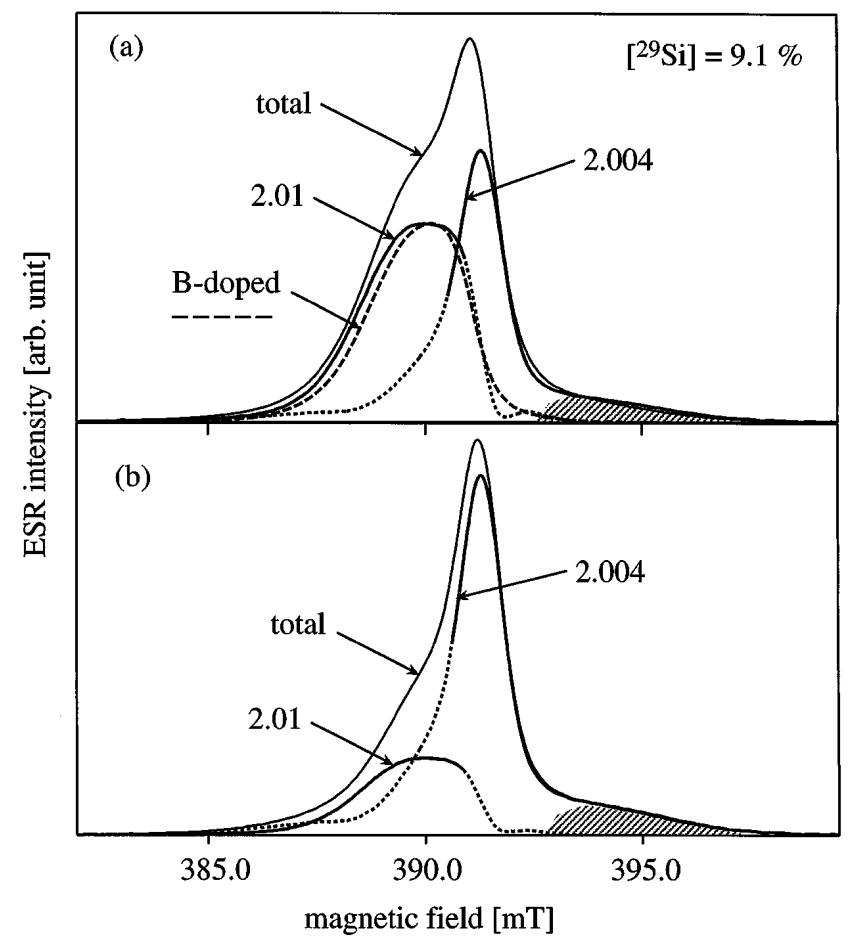

FIG. 3. Deconvoluted spectra of the LESR $(g=2.004,2.01)$ of undoped $a-\mathrm{Si}: \mathrm{H}$. The solid lines indicate the deconvoluted 2.004 and 2.01 spectra with a high reliability in data analyses, while the dashed lines include some ambiguity. (a) Deconvolution of the spectrum (i) in Fig. 2 and the dark ESR spectrum of boron $\left(0.89\right.$ at. \%)-doped $a$-Si:H $\left(T_{s}=200{ }^{\circ} \mathrm{C}\right.$, measured at $50 \mathrm{~K}$ ). (b) Deconvolution of the spectrum (ii) in Fig. 2. Shadow regions show the high-field-side hyperfine structure of ${ }^{29} \mathrm{Si}$. 
$p$ is the number fraction of ${ }^{29} \mathrm{Si}$ atoms in the total $\mathrm{Si}$ atoms. Therefore, the fraction of hf structure for the case of small $N$ and $p \ll 1$ should be $1-(1-p)^{N}$, although this includes the small probability for two or more ${ }^{29} \mathrm{Si}$ atoms to be members of $N \mathrm{Si}$ atoms. The area of the highfield-side hf structure, which should correspond to onehalf of the area of the entire hf structure, was estimated to be $20 \pm 3 \%$ [9] of the total area of the high-field-side spectrum of $g=2.004$,

$$
1-(1-p)^{N}=0.2 \pm 0.03 \text {. }
$$

Using the actual value of $p$ in this experiment, $p=0.091$,

$$
N=2.3 \pm 0.4
$$

is obtained, and therefore it is concluded that the wave function of the 2.004 center spreads mainly over two $\mathrm{Si}$ atoms. The obtained value of $N$ seems to be larger than 2 within some fluctuation. One possible origin might be the contribution from a slight spreading of the wave function on back-bonded Si atoms, but the present experimental data is insufficient for more detailed discussion. We also confirmed this result for the case of $p=4.7 \%$. For $p=1.6 \%$, it was rather difficult to analyze the spectrum because of a small fraction of ${ }^{29} \mathrm{Si}$. In this discussion we assumed that the electron wave function spreads over $N \mathrm{Si}$ atoms uniformly, although the actual wave function may fluctuate among $N \mathrm{Si}$ atoms. However, the fluctuation contributes only to the linewdith of the hf structure and does not affect the above conclusion. Previously, Yamasaki et $a l$. [6] were the first to report the hf structure of LESR spectra in undoped $a-\mathrm{Si}: \mathrm{H}$, and tentatively speculated that the wave function of the LESR spin centers is localized on a single $\mathrm{Si}$ atom simply because a ratio of the area of the $\mathrm{hf}$ structure to a total area of the whole LESR spectrum $(2.004+2.01$ signals $)$ was close to the ${ }^{29} \mathrm{Si}$ content. However, by the present detailed study of the spectrum deconvolution, it has been clarified for the first time that the 2.01 signal does not contribute to the high-magnetic-fieldside hf structure; only the 2.004 signal does.

The isotropic hf splitting which arises mainly from the $3 s$ character in the wave function of the $g=2.004$ center was estimated to be about $7 \mathrm{mT}$ which is almost as large as that of the neutral dangling bond signal $(g=2.004)$ $[2,4]$. Since the isotropic hf splitting due to the $3 \mathrm{~s}$ orbital on ${ }^{29} \mathrm{Si}$ has been theoretically calculated to be $149.0 \mathrm{mT}$ [2], the component of the $3 s$ orbital in the wave function of the 2.004 center is estimated to be about $5 \%$. In contrast to the $g=2.004$ signal, the $g=2.01$ signal apparently does not show any hf structure, from which the isotropic hf splitting of the 2.01 signal was roughly estimated to be below $5 \mathrm{mT}$; in other words, the isotropic hf splitting of the 2.01 signal should be smaller than that of the 2.004 signal. Furthermore, it should be pointed out that the deconvoluted 2.01 signal is quite similar to the dark ESR signal of $g=2.01$ in boron (0.89 at. \%)doped $a-\mathrm{Si}: \mathrm{H}$, as is also shown by the dashed curve in Fig. 3(a). The 2.01 signal of B-doped $a$-Si:H does not show any discernible hf structures, being almost identical to the present deconvoluted 2.01 signal.

So far, two models have been proposed for the origins of the LESR signals. The first one pictures electrons or holes trapped at band-tail states originating from weak $\mathrm{Si}-\mathrm{Si}$ bonds [10]. In an amorphous network, it is quite possible that largely elongated covalent bonds (weak bonds) are present and give rise to energy levels in the tail regions of the conduction and valence bands. In this case, when an electron is trapped in an antibonding state, its wave function is expected to extend over the two $\mathrm{Si}$ atoms associated with the weak $\mathrm{Si}-\mathrm{Si}$ bond. In the second model for LESR centers, electrons and holes are trapped at positively and negatively charged dangling bonds, resulting in the formation of neutral dangling bonds with $g=2.004$ and 2.01, respectively [6,11], which are thought to be similar to the neutral dangling bond observed in the dark. This model essentially originated from the charged defect model for chalcogenide glasses, where negative correlation energy plays an important role [12]. However, a neutral dangling bond state is characterized by an electron localized on a single $\mathrm{Si}$ atom [2-4], which is inconsistent with the present results on the $g=2.004$ LESR signal.

Consequently, the present results suggest that the origin of the LESR signal of $g=2.004$ is electrons trapped at weak $\mathrm{Si}-\mathrm{Si}$ bonds whose antibonding states are located at the conduction-band tail, rather than electrons trapped at positively charged dangling bonds.

Although detailed information on the $g=2.01$ center was not obtained in this study, it is reasonable to consider, by analogy with the $g=2.004$ signal, that it originates from holes trapped in the bonding states of the weak bonds located in the valence-band tail. Katagiri calculated electronic structures of negatively and positively charged weak bonds using the ab initio method, in which a bond length of the positively charged weak bond was shorter than that of the negatively charged one [13]. From that result, it is speculated that the wave function of the 2.01 center may be shifted towards the bond center of the weak bond compared to that of the 2.004 center, resulting in less density of an unpaired electron of the 2.01 center at Si nuclei associated with the weak bond. This might be the reason why the hf splitting of the 2.01 signal is smaller than that of the 2.004 signal.

The deconvoluted signal of $g=2.004$ shows hf splitting of about $7 \mathrm{mT}$, which is similar to the value for neutral dangling bonds $[2,4]$. Using the linear combination of atomic orbitals (LCAO) expansion, the wave function $|\psi\rangle$ of an unpaired electron is written as $|\psi\rangle=\sum_{i} \alpha_{i}\left(\sigma_{i}|3 s\rangle+\right.$ $\left.\pi_{i}|3 p\rangle\right)$, where $|3 s\rangle$ and $|3 p\rangle$ denote the atomic $3 s$ and $3 p$ orbitals of $\mathrm{Si}, i$ indexes all atoms within the extent of the wave function $|\psi\rangle$, and the projection coefficients $\alpha_{i}$, $\sigma_{i}$, and $\pi_{i}$, obey the normalization conditions $\sum_{i} \alpha_{i}^{2}=$ $1, \sigma_{i}^{2}+\pi_{i}^{2}=1$ for all $i$. The experimental value of $7 \mathrm{mT}$ for hf splitting means $\alpha_{1}^{2} \sigma_{1}^{2} \sim 0.05$ for neutral dangling 
bonds $(g=2.0055)$, where an atom of $i=1$ represents a threefold-coordinated $\mathrm{Si}$ atom, and

$$
\alpha_{1}^{2} \sigma_{1}^{2} \sim \alpha_{2}^{2} \sigma_{2}^{2} \sim 0.05
$$

for the 2.004 center, where atoms of $i=1$ and 2 represent $\mathrm{Si}$ atoms associated with the weak bond, respectively. For the case of the neutral dangling bond, Stutzmann and Biegelsen [2] reported that $\alpha_{1}^{2} \sim 50 \%$ and $\sigma_{1}^{2} \sim$ $10 \%$ [4], and the ratio of $3 s$ to $3 p$ character $\left(\sigma_{1}^{2} / \pi_{1}^{2}\right)$ is estimated to be about $\frac{1}{9}$. As compared with strongly localized dangling bonds, for the signal of $g=2.004$, it is reasonable to consider that the densities of the wave function of this center on two Si atoms, i.e., $\alpha_{1}^{2}$ and $\alpha_{2}^{2}$ are rather less than 50\%. Therefore, to satisfy Eq. (3), $\sigma_{1}^{2}$ and $\sigma_{2}^{2}$ should be higher than $10 \%$. Namely, the $3 s-$ to $3 p$-character ratio for the 2.004 center should be higher than that for the dangling bond.

The ratio of $\sigma^{2} / \pi^{2}$ is related to the angle between a dangling (or weak) bond and back bonds, i.e., a ratio of zero means a $\pi$ electron on a $s p^{2}$-network planer structure, while a ratio of $\frac{1}{3}$ means a tetrahedral structure. In the case of the 2.004 center, $\sigma_{1}^{2} / \pi_{1}^{2}$ and $\sigma_{2}^{2} / \pi_{2}^{2}$ should be higher than $\sigma_{1}^{2} / \pi_{1}^{2}$ for the neutral dangling bond. Therefore, it is speculated that the bonding structure of the 2.004 center is closer to a tetrahedral structure than that of the neutral dangling bond which may be characterized as a planar structure since its wave function consists of mostly $p$ character [2-4]. Likewise, the smaller isotropic hf splitting of the 2.01 signal in comparison to that of the 2.004 signal seems to indicate that the atomic configuration of the 2.01 center is closer to a planar structure than that of the 2.004 center [14].

In conclusion, LESR spectra in ${ }^{29} \mathrm{Si}$ enriched $a$-Si:H (9.1 at. \%) were deconvoluted into two signals of $g=$ 2.004 and $g=2.01$ centers by pulsed ESR techniques using the difference in the spin-lattice relaxation time between the two signals. It was pointed out that the hf structure at the high-magnetic-field side of LESR spectra originates mainly from the signal of $g=2.004$. On the basis of careful data analyses it was demonstrated that the wave function of the 2.004 center is spread mainly over two $\mathrm{Si}$ atoms, which suggests that the origin of the 2.004 center is electrons trapped at antibonding states of the weak Si-Si bonds located at the conduction-band tail rather than those trapped at the positively charged dangling bonds.

We thank H. Katagiri and N. Ishii for valuable suggestions regarding the theoretical aspect. This work, partly supported by NEDO, was performed at the Joint Re- search Center for Atom Technology (JRCAT) under the joint research agreement between the National Institute for Advanced Interdisciplinary Research (NAIR) and the Angstrom Technology Partnership (ATP).

*Permanent address: Electrotechnical Laboratory, 1-1-4, Umezono, Tsukuba, 305, Japan.

[1] J. C. Knights, D. K. Biegelsen, and I. Solomon, Solid State Commun. 22, 133 (1977).

[2] M. Stutzmann and D. K. Biegelsen, Phys. Rev. B 40, 9834 (1989), and references therein.

[3] N. Ishii and T. Shimizu, Phys. Rev. B 42, 9697 (1990).

[4] We have also confirmed this conclusion from analyses of echo-detected ESR measurements, which will be published elsewhere.

[5] R. A. Street, D. K. Biegelsen, and R. L. Weisfield, Phys. Rev. B 30, 5861 (1984).

[6] S. Yamasaki, H. Okushi, A. Matsuda, K. Tanaka, and J. Isoya, Phys. Rev. Lett. 65, 756 (1990).

[7] J. Isoya, S. Yamasaki, A. Matsuda, and K. Tanaka, Philos. Mag. B 69, 263 (1994); J. Isoya, S. Yamasaki, H. Okushi, A. Matsuda, and K. Tanaka, Phys. Rev. B 47, 7013 (1993).

[8] When two signals have a constant (= frequencyindependent) width or a constant component in their width, in higher frequency the overlapping of two signals corresponding to different $\mathrm{g}$ values should be reduced. In the present case, as is shown in Fig. 1, there are appreciable frequency-independent contributions to the signal width by small ${ }^{29} \mathrm{Si}$ hf splittings.

[9] The value of $20 \pm 3 \%$ was obtained on the assumption that the tail region of the main peak of $g=2.004$ decays exponentially with respect to the magnetic field. In order to check the validity of this assumption we applied the same procedure to a dangling bond ESR spectrum of the sample enriched by 9.1 at. $\%$ of ${ }^{29} \mathrm{Si}$. As a result, the ratio of ${ }^{29} \mathrm{Si} \mathrm{hf}$ structure to the total signal area was obtained as $9 \pm 2 \%$, which indicates that the assumption is reasonable because the wave function of this spin center is located mainly on a single $\mathrm{Si}$ atom [2-4].

[10] M. Stutzmann, W. B. Jackson, and C. C. Tsai, Phys. Rev. B 32, 23 (1985).

[11] K. Morigaki, J. Phys. Soc. Jpn. 50, 2279 (1981); D. Adler, Solar Cells 9, 133 (1983); H. M. Branz, Phys. Rev. B 41, 7887 (1990).

[12] R. A. Street and N. F. Mott, Phys. Rev. Lett. 35, 1293 (1975); S. G. Bishop, U. Strom, and P. C. Taylor, Phys. Rev. Lett. 34, 1346 (1975); S. G. Bishop, U. Strom, and P. C. Taylor, Phys. Rev. Lett. 36, 543 (1976).

[13] H. Katagiri, Solid State Commun. 95, 143 (1995).

[14] N. Ishii, Solid State Commun. (to be published). 